\title{
Calcineurin Nephrotoxicity
}

National Cancer Institute

\section{Source}

National Cancer Institute. Calcineurin Nephrotoxicity. NCI Thesaurus. Code C114585.

Kidney injury caused by calcineurin inhibitor immunosuppressive therapy which may lead to diminished kidney function. 